ÉGYPTE

monde arabe

\section{Égypte/Monde arabe}

26 | 1996

Mutations

\title{
Les relations État-société en Égypte : restructurer le politique
}

\section{Salwa Ismaïl}

Traducteur : Samia Rizq

\section{OpenEdition \\ Journals}

Édition électronique

URL : https://journals.openedition.org/ema/1476

DOI : 10.4000/ema.1476

ISSN : 2090-7273

Éditeur

CEDEJ - Centre d'études et de documentation économiques juridiques et sociales

Édition imprimée

Date de publication : 30 juin 1996

Pagination : 131-150

ISSN : 1110-5097

Référence électronique

Salwa Ismaiil, « Les relations État-société en Égypte : restructurer le politique », Égypte/Monde arabe [En ligne], 26 | 1996, mis en ligne le 08 juillet 2008, consulté le 07 juillet 2022. URL : http://

journals.openedition.org/ema/1476 ; DOI : https://doi.org/10.4000/ema.1476

Ce document a été généré automatiquement le 7 juillet 2022.

Tous droits réservés 


\title{
Les relations État-société en Égypte : restructurer le politique
}

\author{
Salwa Ismaïl
}

Traduction : Samia Rizq

\section{NOTE DE L'ÉDITEUR}

Original anglais publié dans Arab Studies Quarterly, vol. XVII, n 3, 1995.

1 Au cours de son histoire moderne, l'Égypte a connu nombre de transformations et de changements structurels fondamentaux, de l'âge libéral au socialisme en passant par une phase de capitalisme dirigé, laquelle a conduit à un État des institutions puis à une phase de réforme. Cette dernière tire son nom de la politique qui détermine en grande partie les mutations actuellement en cours sur le plan économique et que l'on espère voir se produire dans la sphère politique.

$2 \mathrm{Si}$, sous le gouvernement de Hosni Moubarak, l'on utilise volontiers le mot de réforme pour désigner les changements accomplis sur le plan économique, ce terme est utilisé avec beaucoup plus de réserve lorsqu'il s'agit du domaine politique. Le régime actuel a, dès le départ, montré une prédilection, dans les discours publics, pour les termes de démocratisation et de libéralisation : al-dimûcrâtiya wa-l-huriya al-'amma (démocratie et libertés publiques). C'est en ces termes que le régime se définit et qu'il détermine ses objectifs politiques et sa ligne de conduite. Les besoins de la rhétorique s'ajoutant aux attentes, internes et externes, l'expression réforme politique est devenue courante dans le discours public.

Dans cet article, je parlerai tout d'abord des initiatives du régime en matière de réforme politique afin de mettre en évidence les limites de la politique de libéralisation contrôlée. Je montrerai ensuite comment, avec la politisation des syndicats professionnels, un nouveau type de restructuration intervient dans le fonctionnement du système. Dans une première partie, je soulignerai brièvement les caractéristiques du régime et les aspects généraux de la crise de l'État égyptien. Dans une seconde partie, 
mon analyse se focalisera sur la configuration de la scène politique elle-même, en prenant comme point de départ l'épisode récent dit du « dialogue national » (al-hiwâr al-watani) dans lequel on peut voir une illustration des efforts déployés par les détenteurs du pouvoir pour maintenir les structures existantes. En troisième partie, les enjeux de ce « dialogue » seront envisagés dans la perspective des restructurations en cours sur la scène politique, restructurations engagées dans la logique de l'émergence de nouvelles forces, en particulier celles représentées par les organisations professionnelles. Dans une dernière partie enfin, je tenterai de préciser la forme et la nature de cette restructuration par rapport au processus de démocratisation annoncé et en référence au concept de société civile.

\section{La politique du régime et la crise de l'État}

4 La politique égyptienne peut être appréhendée à partir d'un ensemble de caractéristiques structurelles déterminant un mode de fonctionnement qui, lui-même, permet de rendre compte de la dynamique des interactions entre les acteurs en présence sur la scène politique ${ }^{1}$. Ces traits structurels sont déterminés par les pouvoirs étendus de l'exécutif, avec à son sommet le président, et un cadre juridique qui assure le contrôle de l'action politique. À l'intérieur de ce cadre, les lois qui régissent la formation des partis et des associations et qui restreignent l'exercice des droits politiques sont les indices du caractère limité de la démocratisation que le régime s'est engagé à mettre en œuvre. Inscrites dans l'ordre juridique, ces limitations ont contribué à l'émergence de schémas de fonctionnement propres au champ politique égyptien. Dans le cadre de cette libéralisation contrôlée et du fait de la prévalence du jeu de facteurs socio-historiques « hérités », les partis politiques se sont révélés de plus en plus inefficients. L'un des principaux aspects de cette configuration réside dans le faible niveau de participation politique: participation réduite aux élections, petit nombre d'adhérents aux partis. Dans ce contexte - et à un moment de crise qui, du point de vue des participants, rendait nécessaire une mobilisation nationale -, il ne semblait y avoir d'autre issue que le recours à un mécanisme ad hoc: le "dialogue national ».

5 Cette initiative du pouvoir égyptien intervient dans un contexte de crise généralisée de l'État, qui se traduit par la difficulté de ce dernier à faire observer certaines de ses lois. Plusieurs indices corroborent cette proposition, dont, notamment, l'augmentation de la corruption en Égypte et son extension à nombre d'aspects des relations et transactions sociales. Le nombre d'infractions commises au sein de la bureaucratie et portées devant les tribunaux administratifs a atteint, en 1993, $56000^{2}$.

6 Par ailleurs, l'état d'urgence étant toujours en vigueur's la police a vu s'accroître ses prérogatives en matière d'arrestation, d'interrogation et de détention. Dans ce contexte, les pouvoirs quasi discrétionnaires dont elle jouit sont mis au service de ce que O'Donnell (1992) appelle la domination privatisée : Les services de la police sont soustraités à des individus ou à des réseaux organisés qui utilisent cette délégation pour subvertir la loi dans les secteurs dépendant de leur autorité et constitués en véritables fiefs.

7 L'État est en lutte contre des sphères de pouvoir pratiquement indépendantes qui jouissent d'un certain contrôle territorial, non seulement hors des grands centres urbains (villages et villes de Haute-Égypte) mais aussi dans les périphéries urbaines du 
Caire. Les quartiers cairotes d'Imbâba ou de 'Ayn Shams échappaient déjà en partie au contrôle du gouvernement avant de devenir, pour les groupes islamistes, un terrain d'action privilégié. Ces communautés informelles, qui ont émergé de manière spontanée, ont fini par constituer, au cœur du Caire et ailleurs, de véritables villages. Elles ont vu le jour au mépris des lois relatives au logement, à la planification urbaine et à la sécurité. La privatisation des espaces publics touchés par la mainmise de ces communautés et de divers secteurs de l'économie informelle a eu pour effet de remettre en question l'autorité de l'État. Dans de nombreux cas, ce dernier a dû faire appel à ses appareils coercitifs pour tenter de s'imposer.

8 Le régime actuel tente de maintenir son emprise en concentrant son action dans la sphère politique, où sont gérés les problèmes posés par l'existence de ce "néopluralisme " que constituent les réseaux de corruption organisés et les sphères de pouvoir indépendantes. Il tente, par le biais de la stabilisation politique, de pallier les insuffisances de l'État.

\section{Le dialogue national : une politique de stabilisation}

\section{Le contexte}

En octobre 1993, après sa prestation de serment pour un troisième mandat, le président Moubarak lance un appel pour la tenue d'une conférence dont l'objectif annoncé est de relancer le «dialogue national $»^{4}$. En examinant le contexte dans lequel cet appel a été lancé, les péripéties de la préparation de cette conférence et enfin sa teneur même, nous nous attacherons, dans cette partie, à faire ressortir les modes de fonctionnement de l'ordre politique existant, en préalable à la description et à l'évaluation du processus de la restructuration du champ politique.

L'appel du président Moubarak survient à un moment où, pour les observateurs et les analystes locaux, le régime et le pays traversent une période de crise ${ }^{5}$. Une crise qui va atteindre son apogée avec l'intensification des activités militantes des groupes islamistes : vers la fin de 1992 et tout au long de 1993, on assiste en effet à une escalade des attaques islamistes contre le régime, attaques qui entraînent des actes de violence coûtant la vie à plusieurs officiers supérieurs, visant les sites touristiques et atteignant leur point culminant dans des attentats contre de hauts responsables de l'État.

11 Les " crimes contre le tourisme " (garâ'im al-siyâha) commis à cette époque visaient, en interrompant les flux de touristes, à déstabiliser l'économie et le pouvoir en privant le pays d'une importante source de revenus. Ce climat d'instabilité était également perçu comme préjudiciable à la politique de réforme économique devant conduire à la privatisation. L'Égypte ne risquait pas seulement de perdre des investissements étrangers, elle risquait aussi une fuite du capital local. Ces attaques, qui portaient atteinte à la respectabilité du régime à l'intérieur, n'ont nullement contribué à revaloriser son image à l'étranger. Une conférence nationale devait donc servir à montrer l'union contre l'extrémisme et à mobiliser les forces politiques "légales » autour du pouvoir en place.

Bien que la teneur du dialogue n'ait pas été clairement définie lors de sa convocation, son objectif général était de discuter des grandes orientations pour l'avenir du pays. Un objectif aussi peu précis et aussi vaste englobait nécessairement la politique de réforme économique concrétisée par les privatisations et la restructuration. En fait, l'idée d'un 
dialogue national figurait déjà dans une étude de l'US AID, un des principaux " supporters » internationaux du programme de réforme économique. Cette étude, qui traitait de l'impact social de la politique économique, plaidait en faveur d'un nouveau pacte social qui permettrait de répartir, sur une base démocratique, le fardeau de la réforme entre les différentes forces sociales en présence (Middle East Report, 1992).

Le dialogue visait à conforter la stabilité du régime, à légitimer ses orientations économiques, mais surtout à récupérer l'initiative politique. En effet, la menace islamiste l'avait rendu vulnérable face aux pressions exercées par l'opposition en faveur d'une réforme. L'échec de la politique de sécurité (al-siyâsa al-amniya) à apporter une réponse adéquate au défi islamiste avait exposé le pouvoir à une série d'attaques axées sur l'inadaptation des structures en place.

Récupérer l'initiative politique s'avérait également nécessaire face aux défis qu'opposaient au régime des institutions légitimes telles que les syndicats et les tribunaux civils. En 1992, les pouvoirs de l'exécutif, concentrés entre les mains du président, avaient rencontré leurs propres limites: en acquittant une majorité d'islamistes lors de procès engagés devant les tribunaux civils, le corps judiciaire affirmait son indépendance, ne laissant au gouvernement que peu de moyens pour régler ses comptes avec ses adversaires ${ }^{6}$. Le président Moubarak ordonna alors que les islamistes soient déférés devant les tribunaux militaires.

De même, face aux mutations intervenues au sein des syndicats professionnels, les contrôles exercés par l'exécutif sur l'Assemblée du Peuple, organe législatif, de même que les restrictions imposées à l'action politique, se révélaient insuffisants. Dès le milieu des années quatre-vingt en effet, un nombre croissant de syndicats professionnels s'étaient imposés comme espaces politiques « alternatifs » ouverts aux activités de l'opposition. La politisation des syndicats s'étant opérée en parallèle avec leur " islamisation ", ces nouveaux espaces politiques étaient une source de difficultés pour le régime. Les élections aux conseils d'administration des syndicats étaient devenues des événements politiques à part entière et avaient acquis une charge symbolique considérable. Pour faire pièce à cette perte de contrôle manifeste, le gouvernement fit adopter la loi $n^{\circ} 100$ relative à l'organisation des élections syndicales? ${ }^{7}$ Je reviendrai plus loin sur les relations État/syndicats et le processus de restructuration pour me concentrer ici sur la question du dialogue national et sur les termes mêmes dans lesquels peut être abordée la question de la réforme politique dans la logique des modes de fonctionnement du régime.

\section{Les acteurs : légaux et reconnus}

Comme nous l'avons souligné dans ce qui précède, le président Moubarak, en invitant au dialogue, prenait l'initiative de l'action politique et ce, en tant qu'incarnation du pouvoir exécutif étroitement lié au parti au pouvoir, le Parti national démocrate (PND). En fait, en l'absence de voies d'accès appropriées permettant l'alternance au gouvernement, il est difficile de séparer le parti au pouvoir du pouvoir exécutif.

17 D'emblée, le régime affirmait sa maîtrise sur la conduite du processus. Si, dans un premier temps, l'invitation au dialogue avait semblé très large, il avait été décidé, peu de temps après, qu'un comité préparatoire serait constitué, dont les membres seraient nommés par le président. L'examen de la liste des membres de ce comité permet d'identifier les «acteurs reconnus » : il s'agit de personnalités en vue dans la sphère de 
l'État et du parti au pouvoir, de dirigeants de l'opposition et de "personnalités publiques ", cette dernière catégorie étant essentiellement composée de personnalités associées au pouvoir et au PND. Sur les quarante membres du comité, on ne comptait que dix leaders de l'opposition: il était inévitable que cette dernière manifeste sa consternation, sans toutefois pouvoir demander une représentation proportionnelle compte tenu de sa faible surface électorale. Le président avait en quelque sorte ramené l'opposition à la portion congrue qui était la sienne. La composition de la conférence elle-même devait reproduire celle du comité préparatoire, mais à une plus vaste échelle.

De leur côté, les partis de l'opposition avaient déjà exprimé leur préférence pour un dialogue entre partis et axé sur les questions politiques, ce qui leur aurait permis une représentation plus importante dans le processus et une voix plus forte dans le débat. Cependant, en rassemblant un grand nombre de "personnalités publiques » et d'« experts », le PND parvenait en quelque sorte à neutraliser son opposition officielle. De la sorte, il traduisait, quoi qu'il en soit, la réalité de la scène politique, une scène où l'inefficacité des partis est problématique. En même temps, le parti au pouvoir se montrait très sélectif quant à l'identification de ceux qui constituaient selon lui les véritables forces politiques : la désignation des partenaires au dialogue était fondée sur leur légitimité, au sens juridique du terme. Par conséquent, malgré sa considérable capacité de mobilisation, l'organisation des Frères musulmans, ne jouissant d'aucun statut juridique, se vit refuser la participation au processus. De la même façon, avec moins de partisans mais pouvant se prévaloir d'une longue histoire d'activisme politique, les communistes auraient pu légitimement revendiquer une place dans le dialogue, mais elle leur fut refusée du point de vue légal.

Dans toutes ces acrobaties, il fallait prendre en compte l'émergence de nouveaux acteurs sur la scène politique et le fait que certaines institutions s'étaient imposées comme nouvel espace politique et comme forum de l'opposition. Compte tenu des bases institutionnelles de la représentation, les syndicats professionnels furent invités à la conférence. Leurs présidents étaient donc au nombre des participants, sans toutefois que cela signifie une reconnaissance de leur rôle politique par le régime. De plus, la plupart des présidents de syndicats entretiennent d'étroites relations avec le parti au pouvoir, soit en tant que membres, soit en tant que ministres ou anciens ministres ${ }^{8}$.

\section{Les conditions de la réforme politique}

20 Les conditions dans lesquelles s'est déroulé le dialogue confirment le mode de fonctionnement qui est celui du régime de l'actuel chef de l'État depuis son accession au pouvoir en 1981: si, depuis cette date, Hosni Moubarak a donné des signes d'ouverture politique à plusieurs reprises, il n'en a pas moins fait régulièrement obstacle à tout changement réel dans la structure politique - ainsi aux élections de 1987 et 1990. En 1987, à la suite d'une décision de la Cour suprême déclarant anticonstitutionnelles les élections législatives tenues en 1984, le président a prononcé la dissolution de l'Assemblée du Peuple. Adoptée à la veille d'une rencontre qui devait regrouper tous les partis et - déjà - discuter des stratégies à mettre en œuvre pour opérer un changement politique, une telle décision constituait une mesure "préventive " (Ebeid, 1988). De la même façon, la dissolution, en 1990, du parlement élu en 1987, une décision de justice ayant également déclaré anticonstitutionnelles les conditions de son élection, donnait l'impression qu'existait une volonté politique de 
redéfinir les règles du jeu et de rajeunir la classe politique (Farag, 1991). Une fois de plus, en rejetant les revendications de l'opposition demandant que soit garantie l'intégrité du scrutin, le gouvernement affirmait sa volonté de garder le contrôle du processus de démocratisation (Farag, idem). À chacune de ces occasions, les décisions du régime sont venues attester de sa volonté de maintenir le statu quo.

21 Lors de la convocation de la conférence du dialogue, on pouvait encore penser qu'il s'agissait d'un pas en avant, s'il n'avait été suivi de plusieurs bonds en arrière... Ainsi, deux mois environ avant la date d'expiration de l'état d'urgence, Hosni Moubarak prorogeait celui-ci pour une nouvelle période de trois ans. La levée de l'état d'urgence, considérée comme une mesure fondamentale pour œuvrer à une véritable libéralisation politique, était une des principales revendications de l'opposition. Par ailleurs, durant la période précédant la conférence, un certain nombre de lois destinées à renforcer le pouvoir de l'exécutif avaient été soumises à l'Assemblée, entre autres une loi modifiant les conditions de désignation des maires et chefs de village et qui prévoyait que ces postes seraient désormais pourvus par voie de nomination et non d'élection. Une proposition d'amendement de la loi sur la désignation aux postes de président des clubs universitaires avait également été déposée pour que ceux-ci soient eux aussi pourvus par nomination.

La composition de la conférence et les modalités de sa préparation et de son déroulement reflètent la carte politique de l'Égypte telle qu'elle a été dessinée par ses dirigeants et traduisent le souci majeur d'éviter tout changement. En tant que mécanisme ad hoc de participation, celle-ci aura joué un rôle minimum. Dans la mesure où les participants étaient choisis par le président et non désignés par les électeurs, sa configuration ne faisait que reproduire celle des structures existantes et les rapports de forces dominants. Le grand nombre d'universitaires et d'experts a contribué à donner au dialogue un caractère technocratique et élitiste plutôt qu'à en faire le support d'expression d'une "identité nationale». Il est intéressant de constater que les différentes forces et classes sociales, en tant que sujets de la participation et de l'action politiques, n'ont pas été mentionnées et que seules les références institutionnelles ont servi à désigner le groupe dont étaient issus les participants: partis politiques, syndicats, universités, médias, centres de recherche. Seules exceptions dans le jeu de ce mécanisme d'identification institutionnelle: les «jeunes » et les «femmes", associés en tant que tels à la conférence.

Par ailleurs, en établissant lui-même l'ordre du jour des débats, le gouvernement s'assurait le contrôle du processus. La question de la réforme politique, entendue comme réforme constitutionnelle, ne figurait pas parmi les thèmes retenus dans cet ordre du jour. Les propositions d'amendements à la Constitution en vue d'instaurer une plus grande liberté politique furent également rejetées. Les principaux thèmes prévus étaient les suivants : la question des quartiers « informels » figurait en première place, suivie des problèmes du chômage, de l'éducation, de l'habitat, etc. Quant aux thèmes retenus par la commission politique de la conférence, ils tournaient autour de quatre axes: la réforme du système électoral; la révision de la législation relative au procureur général socialiste (al-mudda'i al-'âmm al-ishtirâki); la confrontation avec l'extrémisme ; l'extension des pouvoirs de l'Assemblée consultative (majlis al-shûra).

Un tel ordre du jour plaçait les partis de l'opposition devant un dilemme : omettre la question de la réforme politique ou la placer au bas de la liste des priorités, c'était mettre la cohésion de ces derniers à l'épreuve dans la mesure où c'était le seul thème 
sur lequel ils étaient unanimes. En effet, la constitution d'un front uni de l'opposition sur des questions telles que la libéralisation de l'économie ou l'habitat était improbable, compte tenu des divergences idéologiques de ses composantes et des préoccupations de leurs clientèles. En réussissant à diviser les rangs de l'opposition officielle, la stratégie du gouvernement a donc fait preuve d'efficacité. Seul le Wafd semblait mettre comme condition à sa participation au dialogue que la question de la réforme politique et constitutionnelle fût placée en première ligne des priorités de la conférence ${ }^{9}$.

Dans ses recommandations finales, présentées en juillet 1994, le comité met fortement l'accent sur la réforme du système électoral, sur la base de propositions du PND recommandant la mise en place d'un scrutin de liste et d'une représentation proportionnelle - qui semblent approuvées par les participants ${ }^{10}$. Tout en retenant certaines des suggestions émises par les partis de l'opposition en matière de réforme, ces recommandations restent cependant cantonnées dans les limites du discours officiel (celui du régime et celui de l'opposition). Les options avancées sur la question de la démocratisation mettent l'accent sur les moyens et les mécanismes à mettre en œuvre pour ouvrir l'accès au pouvoir - ce qui correspond, en fait, aux revendications convenues de démocratisation du régime et du cadre juridique de l'action politique qui ont été celles de l'opposition tout au long de la dernière décennie.

La focalisation du dialogue sur les structures traditionnelles du politique aboutissait en fait à limiter le jeu des mécanismes de la démocratie aux aspects conventionnels du pluralisme politique, à savoir les élections, le multipartisme et les libertés publiques. Par contre, le système juridique dans son ensemble, aspect pourtant essentiel de l'identité démocratique ou autoritaire de l'État (O'Donnell, 1992), a été ignoré.

\section{Les retombées du dialogue national sur la communauté politique}

Certaines questions relatives au processus politique et à la nature de la communauté politique se sont cristallisées à l'occasion du dialogue, en particulier - en ce qui concerne la recherche d'un cadre viable pour la démocratie - celle des structures traditionnelles de participation. La restructuration de la communauté politique ou la redéfinition de la politique elle-même étaient ainsi à l'ordre du jour. Les partis n'en sont plus les principaux acteurs et de nouvelles figures sont entrées en scène, en particulier les organisations professionnelles et les institutions spécialisées. Ainsi, même si, comme il a été dit, la composition du comité du dialogue n'était peut-être pas représentative de la scène politique, sa dynamique n'en a pas moins eu pour effet de confirmer l'inefficacité des partis.

Par ailleurs, la politisation d'organisations civiles telles que les syndicats n'a pas manqué de soulever des questions sur la nature de la communauté politique. Peut-on pour autant parler d'une restructuration du politique et de l'émergence de nouvelles formes de participation à caractère démocratique ? L'idée d'un dialogue national et le besoin qui s'en est fait sentir indiquent que les structures et les formes conventionnelles d'activités politiques comme, par exemple, la consultation, sont peu appropriées et, selon certains observateurs, non représentatives. L'on a ainsi évoqué le fait que les mécanismes libéraux traditionnels comme les élections ou le parlement ne garantissent pas la représentation populaire. C'est ce qui amène 'Abd al-'Azîm Ramadân, historien et critique politique, à proposer que le parlement soit composé de 
représentants des institutions sociales, des organisations économiques, des syndicats professionnels, des unions de travailleurs et des ONG (Ramadân, 1994).

Ce point de vue contredit la position des partis de l'opposition et plus particulièrement celle du Wafd ${ }^{11}$. L'argumentation soutenue par ce dernier met l'accent sur le caractère institutionnel de la démocratie libérale (élections, libertés, etc.). Basée sur les garanties constitutionnelles, celle-ci est indissociable de ses fondements juridiques, position qui a pour effet de reporter le débat sur la possibilité d'engager des transformations fondamentales dans le cadre de l'actuelle Constitution. Plus important encore est le fait qu'une telle position privilégie une certaine représentation du politique, à la fois institutionnelle et procédurale, qui elle-même constitue une option lourde sur le type de « démocratie » qu'il s'agit de mettre en œuvre. L'une et l'autre position proposent le changement de la structure politique en place: les propositions du Wafd sont l'aboutissement de pratiques et d'idées bien établies dans les «sociétés de la démocratie réelle "; celles de 'Abd al-'Azîm Ramadân sont le fruit de son analyse de la situation politique en Égypte et de sa lecture du dialogue lui-même, et semblent correspondre, dans une certaine mesure, aux nouvelles réalités - par exemple, la politisation croissante des syndicats et des clubs universitaires. La plupart des observateurs de la scène égyptienne considèrent en effet les activités politiques des syndicats comme partie prenante de la société civile $^{12}$ en voie de constitution, une évolution généralement considérée comme positive dans la mesure où elle est perçue comme une avancée de la démocratie associative ${ }^{13}$. Dans ce qui suit, je me propose d'examiner les implications de la restructuration politique à la lumière de cette notion, appliquée à l'étude du cas des syndicats professionnels.

\section{Démocratie associative et restructuration politique}

\section{La politisation des syndicats professionnels}

Depuis le milieu des années quatre-vingt, la dynamique des relations entre l'État et les syndicats a connu de sérieuses transformations. Ceci est particulièrement vrai dans le cas des syndicats d'ingénieurs, de médecins et, plus récemment, dans celui du syndicat des avocats - dans ce dernier cas toutefois, au terme d'une évolution historique particulière. Jusqu'à une époque récente, les syndicats professionnels étaient sous le contrôle de l'État et étaient intégrés à celui-ci tant sur le plan institutionnel que sur le plan organisationnel. Les présidents des syndicats étaient choisis parmi les hauts fonctionnaires et la majorité des membres appartenaient à la fonction publique : ingénieurs travaillant dans des entreprises publiques, médecins des hôpitaux publics et avocats en poste dans des institutions étatiques. Pour faire partie du conseil d'administration d'un syndicat, il fallait être membre de l'Union socialiste arabe qui, souvent, se chargeait de désigner le président dudit conseil. Les syndicats étaient "apolitiques» en ce sens qu'ils n'avaient pas le droit d'exprimer des opinions politiques et que leur présence sur ce plan se limitait à l'affirmation de leur appui au régime. S'il y a eu des confrontations, elles se sont généralement soldées en faveur du gouvernement et ont abouti par la suite à l'affaiblissement des syndicats ${ }^{14}$. Quant au syndicat des avocats, sa longue indépendance a pris fin avec les tendances corporatistes du régime de Gamal Abdel Nasser et il a adopté, au cours de cette période, une position franchement nassérienne. Sous Anouar al-Sadate, les divergences qui opposaient les avocats à la politique gouvernementale les ont amenés à s'engager à plusieurs reprises 
dans de vifs affrontements avec le régime. Il est à signaler que ces derniers revendiquent un rôle politique particulier en vertu de la nature de leur profession, notamment en matière constitutionnelle ou de défense des droits de l'homme. Ils établissent par là une distinction entre le politique au sens général du terme, et le politique dans un sens plus restreint ${ }^{15}$. J'approfondirai ce point plus loin. Pour l'instant, je mécontenterai de mentionner les indices de politisation des syndicats et procéderai ensuite à l'évaluation de ce phénomène par rapport aux notions de participation démocratique, de communauté politique et de société civile.

\section{Les syndicats : un espace politique nouveau}

31 Vers le milieu des années quatre-vingt, les élections syndicales inaugurent une nouvelle phase de la politique syndicale en Égypte. Les candidats aux conseils d'administration des syndicats des médecins et des ingénieurs présentent un programme revendiquant une "solution islamique " (al-islâm huwa-l-hâl) et un "État islamique " (na'am, nurîduha islâmiyya). Parmi les candidats aux élections apparaissent des figures très étroitement liées à l'organisation des Frères musulmans, notamment le médecin Isâm al-'Aryân et l'ingénieur Abû-l-'llâ Mâdi, qui s'étaient tous deux distingués par leur radicalisme dans les années soixante-dix, alors qu'ils étaient membres des Gamâ'ât islâmiyya sur les campus universitaires.

C'est au cours des élections syndicales du milieu des années quatre-vingt (1984 pour le syndicat des médecins, 1987 pour celui des ingénieurs) que les islamistes remportent leurs premières victoires. Le décollage politique progressif des syndicats s'accompagne ainsi de la mobilisation d'une partie de leurs membres en faveur d'une politique islamiste. Les syndicats des médecins et des ingénieurs constituaient un terrain favorable à de telles activités en raison de la prépondérance des étudiants des facultés d'ingénieurs et de médecine dans les Gamâ'ât durant les années soixante-dix. Un deuxième facteur pourrait avoir influencé le cours des élections au syndicat des ingénieurs : les anciens leaders, accusés de corruption, avaient perdu leur légitimité (Qandîl, 1993). Il en a certainement été de même pour les avocats. Les succès remportés par les avocats islamistes tiennent largement au discrédit dans lequel était tombé le groupe qui avait, pendant si longtemps, dirigé le syndicat. En 1989, les avocats ont été entraînés dans une lutte pour le pouvoir au sein du conseil d'administration, ce qui a donné lieu à de nombreuses accusations de corruption. Du coup, lors de la campagne électorale de 1990-91, les avocats islamistes incitaient leurs collègues à voter pour alaydi al-mutawaddi'a (« les mains purifiées par l'ablution»).

Les différents facteurs du succès initial des islamistes dans les syndicats restent cependant à confirmer par l'analyse des premières élections. On peut néanmoins soutenir que, dans un contexte où les partis politiques se sont montrés inefficaces, les syndicats étaient bien placés pour servir d'espace politique de substitution à un courant qui s'était vu refuser l'accès par les voies normales à la scène politique " officielle ». Leurs succès électoraux ultérieurs ont quant à eux été interprétés comme résultant de leur capacité à répondre aux besoins de leurs membres, notamment en fournissant des services sociaux tels que le logement, la prise en charge médicale, les transports, mais aussi en étendant et défendant les intérêts professionnels des membres en matière de monopoles de compétences, de pensions, de codes déontologiques ${ }^{16}$. 
34 L'éventail des intérêts poursuivis par les syndicats ne se limite cependant pas aux avantages professionnels cités ci-dessus. Sous la direction des islamistes, les syndicats ont également fait des incursions dans la politique nationale et internationale ${ }^{17}$. Le syndicat des médecins a ainsi constitué une commission humanitaire d'aide aux combattants d'Afghanistan et, plus récemment, s'est engagé dans le soutien aux musulmans bosniaques. Également impliqués dans des activités politiques internes, ces syndicats ont apporté leur appui à l'opposition lors du boycott des élections générales de 1990, en rendant public un communiqué sur la question. À ce propos, l'engagement de leurs dirigeants dans les activités des partis mérite d'être signalé. En 1987, les membres éminents des conseils syndicaux se sont lancés dans la campagne électorale pour soutenir les candidats du tahâluf (alliance). Les syndicats ont pris publiquement position en faveur des revendications de l'opposition concernant la réforme constitutionnelle et la levée de l'état d'urgence.

En somme, les syndicats occupent des espaces autrefois réservés à l'État, comme celui des services sociaux et, peu à peu, ils occupent également le vide laissé par les partis. Quelles sont les conséquences d'un tel changement? Si l'on en croit certains signes, la politisation des syndicats aurait eu des effets désintégrateurs, comme en témoignent les derniers événements survenus au syndicat des avocats. Les élections au conseil d'administration se déroulent désormais sur des bases partisanes. Chaque parti politique présente ses candidats, des blocs et des alliances se forment, les Frères musulmans coordonnent les candidatures de leurs membres ${ }^{18}$. Le bloc islamiste affronte le bloc nationaliste et les partisans de la laïcité. Les élections deviennent donc l'arène d'une concurrence politique entre le parti au pouvoir et les partis de l'opposition. Les élections se déroulent à la manière de celles des partis, chacun essayant de regrouper ses adhérents. Plus habiles en matière d'organisation, les islamistes assurent le transport (par bus) de leurs partisans jusqu'aux bureaux de vote. Alors que les uns veulent transformer l'ordre des avocats en organisation islamique, les autres se battent pour lui préserver son identité nationale et laïque.

Les luttes internes se traduisent parfois par des accusations de corruption pouvant aboutir à l'ajournement des élections, comme cela s'est produit en 1993 au Caire et à Giza. En réponse, le conseil d'administration, à majorité islamiste, a accusé ses opposants de diffamer l'institution et menacé de les radier du syndicat des avocats. Au cours de développements ultérieurs, les avocats dissidents ont élaboré un projet de dissolution du syndicat et proposé de nouvelles règles d'organisation ${ }^{19}$.

En mai 1994, à la suite du décès d'un avocat islamiste détenu par la police, le syndicat des avocats entre en conflit avec le gouvernement. Dans un premier temps, les avocats cherchent à former un front uni en lançant un mot d'ordre de grève et une manifestation. Évoquant l'état d'urgence, le gouvernement intervient pour les en empêcher, la police arrosant les manifestants de bombes lacrymogènes tandis que plusieurs avocats sont arrêtés, certains d'entre eux à leur domicile. Cependant, au cours des négociations visant à mettre un terme au conflit, le front se brise : au terme d'un accord conclu entre le gouvernement et le camp des islamistes, les sympathisants des Frères musulmans sont relâchés alors que les autres sont toujours incarcérés. Consterné, le président du syndicat du Caire entame une grève de la faim, ce qui contribue à la libération des autres accusés. Le conflit avec le gouvernement portait sur des thèmes d'intérêt national (les droits de l'homme), mais, de nouveau, l'action des 
acteurs était motivée par une politique partisane. La politique au sens large du terme se trouvait ainsi sacrifiée à une définition plus étroite.

Dans cette conjoncture, l'activité politique des syndicats soulève la question plus large de celle des associations civiles et souligne la nécessité de distinguer les intérêts civiques des intérêts professionnels, ainsi que les lieux appropriés à la représentation de chaque type. Cela dit, il faut reconnaître que les deux types d'intérêts peuvent parfois se recouper, s'opposer, coïncider... Il est intéressant de souligner à ce propos que certains avocats, dans leur appréhension du politique, distinguent le sens général, c'est-à-dire non partisan, du terme, et un sens plus restreint, c'est-à-dire partisan.

Le modèle de restructuration politique proposé et les reconfigurations qui se mettent effectivement en place sur la scène politique en Égypte appellent quelques réflexions d'ordre à la fois théorique et pratique. La satisfaction avec laquelle cette restructuration a été accueillie dans le milieu des intellectuels et des observateurs est fondée sur la foi de ceux-ci en la démocratie associative en tant que forme de représentation plus adéquate. Selon ce point de vue, la réforme devrait porter d'abord sur le plan social plutôt que sur le plan politique traditionnel (système électoral, parlement, partis). Cependant, à supposer que les réformateurs ou les militants aient à l'esprit une certaine notion de la démocratie associative, on pourrait soutenir qu'ils se sont écartés de leur objectif, et même que cet écart était quasi inévitable. Pour étayer cet argument, j'exposerai tout d'abord les présupposés de la démocratie associative et je tenterai de voir en quoi elle diverge de l'idéal de la démocratie. Je montrerai ensuite le caractère pratique de ces présupposés et leur caractère plausible du point de vue de l'actualisation et du fonctionnement de la démocratie.

L'activité associative et la revendication du droit de représentativité requièrent en effet certaines conditions. Les associations ont des caractéristiques bien déterminées, indicateurs selon lesquels sont réglementés aussi bien leurs rapports avec l'État que leur rôle dans la société. L'étendue des intérêts d'une association est conventionnellement liée au taux d'adhésion. Dans le cas où l'organisation du groupe est fondée sur le statut de l'individu dans la société (sa profession par exemple), le domaine de représentation de l'association se définit en termes d'intérêts fonctionnels liés à l'activité de ses membres ou à leur statut. Les possibilités d'extension de l'association dépendent donc de l'intégration des individus occupant ces postes ou exerçant ces activités. Les conditions d'adhésion sont donc plus restrictives que dans «les organisations au territoire plus étendu » comme les partis politiques. L'objectif principal de ces associations étant de défendre les intérêts professionnels de leurs membres, c'est en termes de nombre d'adhérents et d'étendue des intérêts que sont définis leurs traits caractéristiques, et c'est en fonction de ces mêmes critères que la nature et les activités des organisations sont réglementées.

Il existe en outre un facteur en corrélation avec ce qui précède : celui du caractère public de l'organisation, trait fondamental de l'action et de l'engagement politiques. Or, les associations semi-publiques, organisations qui imposent des restrictions à l'adhésion, ne répondent pas aux conditions permettant une représentation et une participation égales. Les restrictions que prévoit une association professionnelle sont nécessairement établies selon le mode de fonctionnement qui lui est propre, et c'est précisément à cause de ces restrictions que l'association n'est pas accessible à tous les citoyens. 

les principes de la participation démocratique, et en particulier la corrélation entre l'étendue des intérêts, le nombre d'adhérents et le caractère public de l'organisation en tant que facteurs qui déterminent la représentativité et l'appropriation d'espaces propres à l'action politique. Se posent ensuite les questions relatives à la politisation du social. À ce sujet, la théorisation actuelle s'est développée par référence au contexte est-européen. L'enthousiasme suscité par les institutions de la société civile et leur rôle dans l'effondrement du système communiste s'est exprimé dans les reformulations du politique à partir de l'expérience est-européenne (Walzer, 1991). On assiste ainsi à un renouveau de la foi dans le civisme, dans la solidarité sociale, dans la politique et la démocratie quotidiennes. Je n'ai pas l'intention de reproduire ici les arguments en faveur de la société civile ou de développer l'idée de société civile. Je me contenterai d'en exposer trois caractéristiques telles qu'elles apparaissent dans le modèle esteuropéen. Il a cependant été prouvé que, dans des circonstances différentes, ces caractéristiques ne sont plus aussi pertinentes. Ce sont les suivantes : la formation de grandes alliances, la «politique apolitique » et l'opposition au gouvernement. Comme le fait remarquer Kiss, les grandes alliances se brisent dès l'arrivée au pouvoir des leaders dissidents; la "politique apolitique » entrave la formulation de politiques; et lorsque les forces de l'opposition accèdent au pouvoir, l'opposition elle-même devient obsolète (Kiss, 1992).

43 Si la société civile ne peut pas constituer une alternative à la politique, elle ne peut fonctionner que sous certaines conditions. L'appropriation des espaces publics en tant que domaines de liberté servant de tampons face à l'État dépend d'un concept politique au sens général du terme, utilisé par certains avocats égyptiens, à savoir la politique non rattachée à un parti, ou " politique apolitique ». Néanmoins, ce qui s'est produit en Égypte, c'est l'«idéologisation» des espaces du domaine public au-delà de leur «fonctionnalité ». En tant que telles, les grandes alliances et la politique consensuelle sont exclues.

44 La pratique quotidienne de la démocratie n'est possible que si les intérêts contestés s'inscrivent dans les limites de la conformité aux conditions d'adhésion au groupe. Dans le même ordre d'idées, l'extension des intérêts et la mise en place d'une politique délibérante à caractère public engageant la collectivité deviennent des conditions nécessaires à la participation démocratique. Autrement dit, la clé de la politique quotidienne réside dans l'éventail des décisions susceptibles d'être adoptées.

\section{Conclusion}

La conception officielle de la réforme telle qu'elle se dégage de la dynamique du dialogue se cristallise autour des changements survenus dans les structures traditionnelles de la politique. De ce point de vue, le régime mène une politique mêlée de compromis et de répression - tenir les rênes de l'initiative politique et faire observer les règles du jeu au nom de la légalité et au détriment de la légitimité. Cette forme du politique est maintenant remise en question par une restructuration qui n'est plus du seul ressort du gouvernement. La politisation des syndicats représente l'appropriation d'une autre scène où l'action est menée par de nouveaux acteurs, apparemment plus compétents que les politiciens des partis. La situation à laquelle on aboutit alors est considérée comme le triomphe de la démocratie associative. Cela pose cependant 
plusieurs questions relatives à la nature de la participation démocratique et de la communauté politique.

\section{BIBLIOGRAPHIE}

ABD ALLAH A., «Le cadre de la participation en Égypte », Démocratie et démocratisation dans le Monde arabe, Dossiers du Cedej, Le Caire, 1992.

BIANCHI R., « The Strengthening of Associational Life and its Potential Contribution to Political Reform in Egypt, Turkey and Lebanon ", paper presented at the conference on Political Liberalization and Democratization in the Arab World,McGill University \& Université de Montréal, $7-8 / 05 / 93$.

COHEN, JOSHUA \& ROGERS, « Secondary Associations and Démocratie Govemance », Politics and Society 20,1992, p. 391-472.

DESSOUKI A. H., « L'évolution politique de l'Égypte : pluralisme démocratique ou néoautoritarisme? ", Maghreb-Machrek127, 1er trim. 1990, p. 7-16.

EBEID M. M., « Le rôle de l'opposition officielle en Égypte », Maghreb-Machrek119, 1er trim. 1988, p. 5-24.

FARAG I., « Le politique à l'égyptienne : lecture des élections législatives », Maghreb-Machrek133, $3^{\text {e }}$ trim. 1991, p. 19-33.

IBRÂHÎM H. T., « Binâ' al-mujtama' al-madani : al-mu'ashirat al-kamiya wa-l-kayfiya » (La construction de la société civile : indicateurs qualitatifs et quantitatifs), Al-mujtama' al-madani fi-lwatan al-'arabi wa dawru-hu fi tahqîq al-dimûcrâtiya(La société civile dans le monde arabe et son rôle dans l'élaboration de la démocratie), Markaz dirâsât al-wihda al-'arabiya, Beyrouth, 1992.

IBRÂHÎM S. E., « Al-Muqaddima » (Introduction), Al-mujtama' al-madani wa mustaqbal aldimûcrâtiya fi-l-watan al-'arabi(La société civile et l'avenir de la démocratie dans le monde arabe), Centre Ibn Khaldûn, Le Caire, 1993.

ISMAÏL S., « The Civil Society Concept and the Middle East: Questions of Meaning and Relevance », paper presented at the Annual Meeting of the Canadian Political Science Association, Université de Calgary, 12-14/06/1994.

KISS E., « Democracy Without Parties ? » Dissent, 2e trim. 1992, p. 226-231.

QANDIL A., " Al-tayâr al-islâmi fi-l-niqâbât al-mihaniya al-misriya » (Le courant islamiste dans les syndicats professionnels égyptiens), Al-islàm wa-l-hadâtha : ru'ya islâmiya wa gharbiya 'an Misr wa Turkiya(Islam et modernité : visions islamistes et occidentales sur l'Égypte et la Turquie), Yafa Center for Studies and Research, Le Caire, 1993.

MiddleEast Report22, nov.-déc. 1992, p. 179.

MOORE H. C, Les syndicats professionnels dans l'Égypte contemporaine : l'encadrement de la nouvelle classe moyenne, Maghreb-Machrek64, 3e trim. 1974, p. 24-34. 
O'DONNELL G., « On the State, Democratization and Some Conceptual Problems: A Latin American View with Glances at Some Postcommunist Countries », World Development21, 1992, p. 1355-1369.

RAMADAN A., « Lughz al-hiwâr al-watani » (Le puzzle du dialogue national), al-Ahrâm, 25/06/94.

SA'ÎD M. S., « Al-taghyîr wa-l-wifâq al-watani. » (Changement et consensus national), al-Ahrâm, $30 / 07 / 93$.

SAYYID M. K., « A Civil Society in Egypt? », Middie East Journal47, 2e trim. 1993, p. 228-242.

SCHMITTER P. C, « The Irony of Modem Democracy and Efforts to Improve its Practice », Politics and Society20, 1993, p. 507-512.

SPRINGBORG R., « Professional Syndicales in Egyptian Politics, 1952-1978 », International Journal of Middie East Studies9, 1978, p. 275-295.

WALZER M., « A Better Vision: The Idea of Civil Society », Dissent, 2e trim. 1991, p. 293-304.

Journaux et magazines : al-Ahrâm, al-Ahâli, al-Wafd, al-Sha'b : octobre 1993 à juin 1994; Rose alYûsuf.

Autres souces : Magallat al-Muhandisîn(Revue des ingénieurs), 1989, 1991.

\section{NOTES}

1. Pour l'étude de ces caractéristiques, voir, dans la bibliographie ci-après, Dessouki (1990) et 'Abd Allah (1992). Pour les modes de fonctionnement du régime, voir Farag (1991) et Ebeid (1988). 2. Al-Ahrâm, 24/02/94.

3. L'état d'urgence, décrété dès après l'attentat qui a coûté la vie au président Anouar al-Sadate, n'a jamais été levé malgré la demande répétée des partis de l'opposition et des organisations de défense des droits de l'homme (N.D.L.R.).

4. La Conférence du dialogue national s'est tenue du 26/06 au 10/07/94.

5. Voir al-wafd al-Ahâli entre décembre 1993 et juin 1994. En fait, l'idée d'un dialogue national avait été lancée par les intellectuels dès 1993 (voir Sa'îd, 1993).

6. En août 1993, par exemple, un tribunal civil a acquitté 24 islamistes inculpés dans l'assassinat de l'ancien président du Parlement, Rif'at al-Mahgûb.

7. Conformément à cette loi, un quorum de $50 \%$ des membres de l'assemblée générale du syndicat est requis pour que soient validés les résultats du scrutin. Dans l'éventualité d'un second tour, le quorum requis est de $30 \%$, faute de quoi un conseil d'administration provisoire composé de magistrats est désigné, puis chargé d'organiser des élections où le quorum pourra être atteint et $d^{\prime}$ ' expédier les affaires courantes».

8. $\mathrm{Y}$ compris dans les organisations syndicales contrôlées par le courant islamiste, où celui-ci a choisi, pour des raisons "tactiques", de se réserver les postes exécutifs - secrétaire général, trésorier... -tout en maintenant au poste de président des personnalités choisies pour leur " entregent».

9. Le Wafd et les nassériens se sont retirés à l'issue de la première séance du comité préparatoire qui s'est tenue le 01/06/94.

10. Les élections législatives de novembre 1995 ne s'en tiendront pas moins sous le régime des candidatures individuelles et du scrutin majoritaire.

11. Voir al-Wafd de décembre 1993 à juillet 1994, et plus particulièrement la rubrique de Saïd 'Abd al-Khâliq.

12. Le concept de société civile est de plus en plus utilisé comme outil d'analyse des pays arabes. Les organisations civiles et les activités qu'elles exercent occupent une place centrale dans ces 
études ; voir, par exemple, Al-Sayyid (1993), Ibrâhîm (1992), Ibrâhîm (1993) et Qandîl (1993). J'ai analysé l'utilisation qui est faite de ce concept dans un ouvrage récent (Ismâ'îl, 1994).

13. Le terme de démocratie associative fait l'objet de définitions diverses et, dans une large mesure, contradictoires. Tantôt il englobe la notion d'activisme sociétal séparé de l'État et s'insère, en ce sens, dans le cadre de l'approche de la société civile. Tantôt il est utilisé dans le sens particulier que lui attribue l'approche corporatiste, selon laquelle la démocratie associative est fortement dépendante du pouvoir étatique. Les variantes du corporatisme associatif sont énoncées dans Cohen et Rogers (1992) et dans Schmitter (1992). Les propositions relatives à la démocratie corporatiste ont été adoptées en référence à l'ouvrage de Bianchi sur le MoyenOrient. La critique formulée dans la présente section s'applique davantage au premier sens de ce terme, dans la mesure où le second met l'accent sur le rôle régulateur de l'État. Il est à noter qu'il n'existe pas de définition précise du concept de société civile et que la question des relations État-société a elle aussi fait l'objet de diverses formulations (Ismâ'íl, 1994).

14. Pour un récapitulatif de ces confrontations, voir Moore, 1974. Voir également Springborg, 1978.

15. Cette distinction a été soulignée au cours d'entretiens menés avec M. Muhammad 'Abd al-'Aziz, bâtonnier de la branche cairote du syndicat, et d'autres avocats représentant différentes tendances politiques au sein de celui-ci (août 1994).

16. Les syndicats des médecins et des ingénieurs ont lancé des projets de logement et organisé la vente de biens de consommation hautement prisés tels qu'électro-ménagers, voitures... (Qandîl, 1993) ; voir aussi Magallat al-Muhandisîn (Revue des ingénieurs), par exemple les numéros des premiers trimestres des années 1989 à 1991.

17. Les partisans de l'activisme politique des syndicats se réfèrent à la Constitution, qui garantit un tel droit, ainsi qu'aux règlements des différents syndicats. Ils s'appuient notamment sur les dispositions qui affirment le rôle national assumé par les syndicats dans les affaires de la nation.

18. Rose al- Yûsuf, 11/04/1994.

19. Rose al-Yûsuf, 06/06/1994.

INDEX

Mots-clés : État, politique

\section{AUTEURS}

\section{SALWA ISMAÏL}

CDSA, McGill University, Montréal 